\title{
Scleral Fixation of Intraocular Lens in Aphakic Eyes without Capsular Support: Description of a New Technique
}

\author{
Umesh Chandra Behera (D) \\ Pratima Singh Thakur (1) \\ Retina-Vitreous Service, L V Prasad Eye \\ Institute, Bhubaneswar, 75I024, Odisha, \\ India
}

Purpose: To study the efficacy and safety of a modified trans-scleral intraocular lens (IOL) fixation technique in aphakic eyes when performed by ophthalmologists in training.

Methods: The study was conducted in an institutional setting that included 43 surgeries performed by surgeons training in small incision cataract surgeries. The data were analyzed for stability and position of IOL, refractive changes, best-corrected vision, and associated complications.

Results: Mean age of the subjects was $53.8 \pm 18.5 \mathrm{yrs}$ (range 6-81yrs). Surgical aphakia (58.14\%) was the most common cause. The corrected distance visual acuity improved significantly at six weeks $(\mathrm{p}=0.0003)$. The mean residual spectacle correction was +0.74 $\pm 1.2 \mathrm{D}$ spherical equivalent (cylinder $-1.6 \pm 1.5 \mathrm{D}$ at $84 \pm 50^{\circ}$ ) at the 6th-month follow-up $(24.35 \pm 6.71 \mathrm{wks})$. Lens tilt on ultrasound biomicroscopy (kappa $0.762 ; \mathrm{p}<0.001)$ and the IOL centration (kappa $0.411 ; \mathrm{p}=0.001$ ), assessed by two independent masked observers, were satisfactory at the 6th-month visit. Transient postoperative vitreous hemorrhage was the most common complication (46.5\%). Cellular deposits on the IOL surface (18.6\%), cystoid macular edema (11.6\%), subconjunctival haptic exposure (4.66\%), and haptic slippage $(2.33 \%)$ were the other complications.

Conclusion: This method of trans-scleral IOL fixation is an effective rescue procedure for eyes with deficient capsular support when ophthalmologists perform in training.

Keywords: aphakia, intraocular lens, posterior capsular rent, trans scleral lens fixation, lens deposits

\section{Introduction}

Intraocular lens (IOL) implantation is the standard of care after senile and pediatric cataract surgery. There is rarely an occasion where a primary IOL implantation is contraindicated. But the possibility of leaving the eye aphakic is not ruled out either - in complications of cataract surgery such as posterior dislocation of lens and significant posterior capsular rent - especially by surgeons in training. These events render the eye unsuitable for a primary IOL implantation. Spectacle correction, contact lens, or a secondarily implanted IOL are the rehabilitative options. Past studies have shown that vision-related quality of life is superior in people with IOL implantation than aphakic spectacle correction. ${ }^{1}$ The technique of secondary IOL implantation in an aphakic eye is placing the IOL in the anterior chamber, fixing to iris, or securing to the sclera with sutures. ${ }^{2}$ But these procedures suffer from complications such as postoperative iritis, late dislocation/tilting of IOL and
Correspondence: Umesh Chandra Behera Retina-Vitreous Service, L V Prasad Eye Institute, Patia, Bhubaneswar, 75I024, Odisha, India

Tel $+9 \mid-674-3987100$

$\mathrm{Fax}+91-674-3987130$

Email umeshcbehera@gmail.com 
pupillary distortion (exclusive to iris fixated lenses), secondary glaucoma, and corneal endothelial decompensation (exclusive to looped anterior chamber IOL), which limit their acceptance. ${ }^{3,4}$

Currently, implantation of a three-piece intraocular lens (IOL) with exteriorization of the haptics through a scleral opening and burying it in scleral pockets - the technique of trans scleral IOL fixation - is favored more than other approaches. ${ }^{5-8}$ There is no published level I evidence though the newer sutureless scleral fixation of (secondary) IOL is the most practiced procedure today. ${ }^{9,10}$ Since the description by Gabor and Pavlidis, ${ }^{5}$ many modifications have been proposed to simplify the technique and improve the IOL stability. Experienced anterior and posterior segment surgeons generally perform the surgeries. To improve the acceptability among beginners, we simplified the method of securing the haptic of rigid IOLs in the scleral pocket by bending its tip. Ophthalmologists attempted the technique while they were in the process of learning cataract extraction. The study analyzed the safety and stability of the procedure.

\section{Methods}

Forty-five patients presenting at a tertiary eye care facility with inadequate capsular support for IOL implantation were offered this modified scleral fixation surgery to implant the IOL between August 2018 and January 2019.

We did a pilot study on the initial 5 cases to assess the lens stability and safety. Based on the encouraging results, we extended it to 40 more patients for study completion. We clubbed the data of both cohorts for analysis. We set the visual acuity efficacy endpoint at 6 weeks and safety assessment endpoint at six months. The study adhered to the tenets of the Declaration of Helsinki. The institutional ethics committee of L V Prasad Eye Institute, Bhubaneswar, India (IEC code 2018-113-IM-22) approved the study. The institutional ethics committee audited the study conduct and the outcome. The authors (UCB and PST) performed all the surgeries after obtaining written informed consent from all study participants; the parents or the legal guardians provided the consent for minors. Both the surgeons were in training for small incision cataract surgery while attempting this scleral IOL fixation technique.

Principal eligibility criteria included aphakic eyes without capsular support secondary to surgical complication/ trauma and spontaneous/traumatic dislocation of lens/IOL. Eyes with scleral thinning, children younger than 4 yrs (as there is continued eye growth till that age), patients with poor visual potential (eg, scarred macula secondary to trauma, optic atrophy), and follow up of less than one year were excluded.

\section{Surgical Technique}

The surgical modification included construction of two lamellar uniplanar scleral pockets measuring $3-4 \mathrm{~mm}$ in length, oriented opposite to each other at 3 and 9 O'clock at $1.5 \mathrm{~mm}$ from limbus using a 23G (Alcon, Ft Worth, TX, USA) microincision vitrectomy trocar after a limited peritomy (Figure 1A and B). The vitreous cavity was accessed with sclerotomy at the open end of the scleral pocket. A three-piece rigid $6 \mathrm{~mm}$ optic PMMA-IOL with polypropylene haptics (Aurolab B3602, Madurai, India) was introduced through a superior triplanar sclero-corneal tunnel (Figure 1C), and the leading haptic was negotiated into the lumen of a bent 26G hypodermic needle (Figure 1D and $\mathrm{E}$ ) in the anterior chamber. A silicone stopper was used to immobilize the externalized haptic. The pulled-out haptics were held a millimeter from the tip by a $23 \mathrm{G} \mathrm{IOL}$ holding forceps (Epsilon, Chino, CA, USA) and were forced into the pre-fashioned scleral pocket, deforming the haptic tip to a bend of $60-90^{\circ}$. This crucial maneuver kept the haptic anchored to the scleral pocket and prevented slippage (Supplemental Video).

\section{IOL Selection}

We intentionally chose a rigid three-piece polymethyl methacrylate IOL as it is used mostly by residents while in training in India. The other reason was to test the viability of tip bending crucial to this technique. We preferred a larger optic diameter to reduce the risk of possible postoperative IOL decentration and decrease the shearing forces on the sclera. ${ }^{11}$ The IOL power was reduced by a diopter from the effective lens power to correct for a possible postoperative myopic shift as recommended for sulcus fixated IOLs. ${ }^{12}$

All the eyes were imaged with ultrasound biomicroscopy (Marvel UBM; Appasamy associates, Chennai, India) and scanned by optical coherence tomography (OCT; DRI OCT Triton Plus; Topcon, Tokyo, Japan) preoperatively and at 6 week, 12 week, 3 month and 6 month visits. The OCT at later visits was as per the surgeons' discretion when CME was suspected clinically or an unexplained drop in vision. Two independent observers judged the IOL centration postoperatively on anterior segment photographs and the IOL tilt on UBM. The pictures were taken after full pupil dilatation 


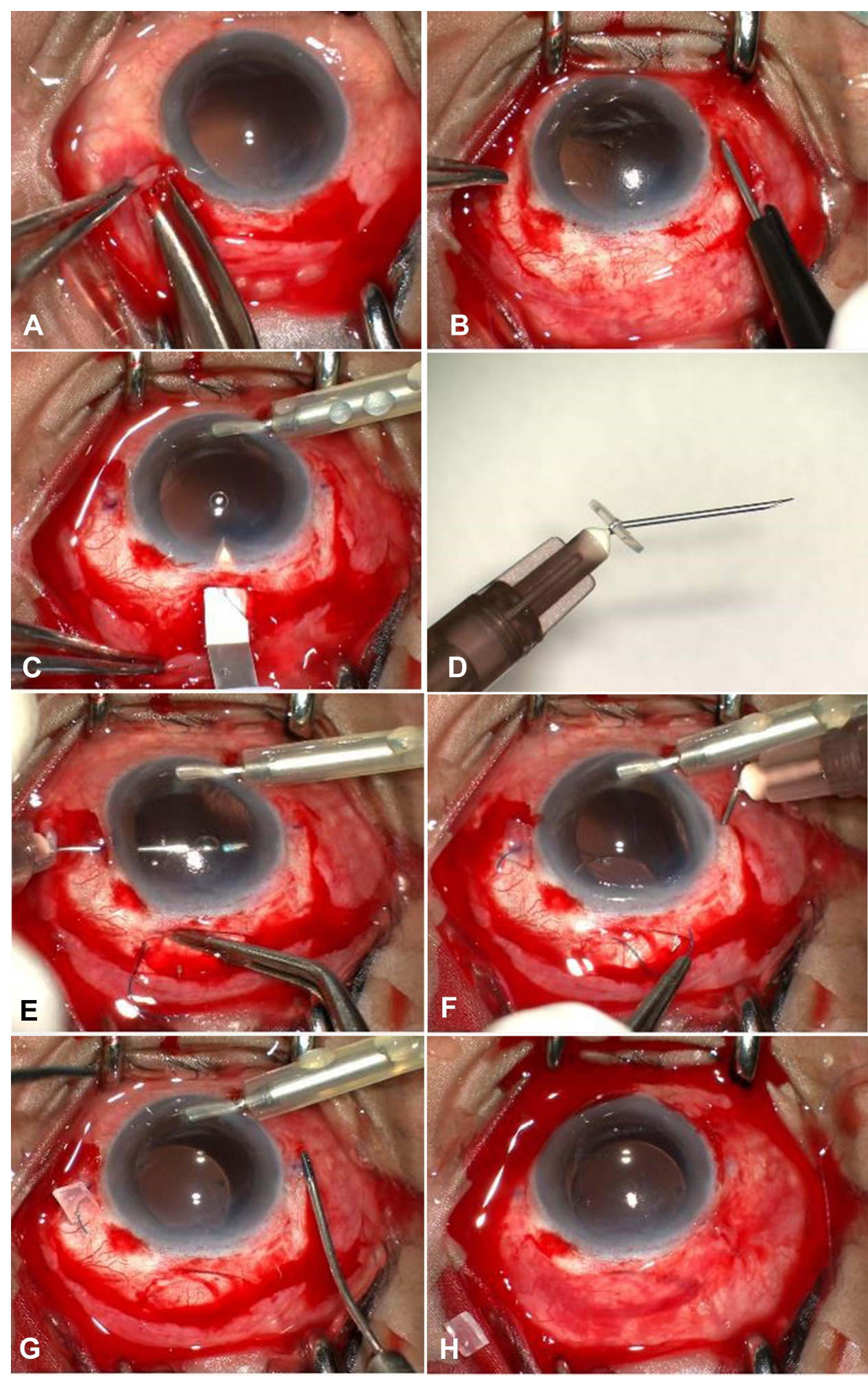

Figure I After limited conjunctival peritomy (A) 2 diagonally opposite scleral tunnels are fashioned and opened to the vitreous cavity by sclerotomy (B). A superior sclerocorneal incision is made $(\mathbf{C})$. A bent hypodermic needle $(\mathbf{D})$ receives the leading haptic in the anterior chamber $(\mathbf{E})$ and the externalized haptic is sleeved by a silicone element for haptic stabilisation. The externalised (F) haptics are held at a measured distance from the tip with forceps and pocketed into the scleral tunnel (G) to bend the tip. Lens centration ensured at the end of the procedure $(\mathbf{H})$. 
to reveal the IOL optic edge. If the ratio of the limbus to the IOL-optic-edge distance on either side (at the horizontal axis running through the center of the cornea) varied between $0.5-1.5$, it was considered centered. The eyes in which the optic edge was not visible, an imaginary arc was created on the picture to correspond to the obscured edge of the IOL. The IOL optic angle against the iris plane of more than 15 degrees was considered a tilt on UBM.

The UBM examination was done at the six-month followup visit for all; a period deemed adequate for any lens position complications to occur. UBM, a contact examination, was avoided in the early postoperative period because the cohort included open globe injury, penetrating keratoplasty, and large corneoscleral tunnel for rigid IOL implantation.

The examination at each visit also included a comprehensive eye examination. Routinely, these included measurement of presenting vision, bestcorrected visual acuity (BCVA), subjective refraction, applanation tonometry, slit-lamp biomicroscopy, and indirect ophthalmoscopy.

\section{Statistical Analysis}

Categorical variables were presented in numbers, and percentages $(\%)$ and continuous variables were presented as mean \pm SD and median. Normality of data was tested by Kolmogorov-Smirnov test. If the normality was rejected, then a non-parametric test was used. Visual acuity scores were compared using Mann-Whitney Test (as the data sets were not normally distributed) and paired $t$-test/Wilcoxon signed-rank test was used for comparison between preoperative and postoperative vision. Qualitative variables were correlated using the Chi-Square test/Fisher's Exact test. Finally, an inter-rater kappa agreement was used to find out the strength of agreement between the two observers. A p-value of $<0.05$ was considered statistically significant. The data was entered in MS EXCEL spreadsheet and analyzed using Statistical Package for Social Sciences (SPSS) version 21.0.

\section{Results}

Data analysis of 43 of 45 subjects who fulfilled the entry criteria was done. Two recruited patients did not meet the follow-up criteria and hence were excluded from the analysis. The mean age of the 31 males (72.1\%) and 12 females $(27.9 \%)$ was 63.8 yrs. Causes of aphakia were surgical (58.14\%), traumatic dislocation of the lens $(32.5 \%)$, and spontaneous posterior dislocation of the lens $(9.3 \%)$. The surgeons self-managed seven out of 43 eyes $(16.3 \%)$ in training with modified SFIOL technique when capsular complications were encountered during cataract extraction. The mean duration of aphakia was $5.22 \pm 9.05$ months (range 0 to 60 months). Two of the eyes were on topical anti-glaucoma medication (AGM) before the surgery. Both of them had lens dislocation secondary to trauma. The mean intraocular pressure was $13 \pm 5 \mathrm{mmHg}$ on postoperative day one, $14.6 \pm 3.5$ $\mathrm{mmHg}$ at 6-week-postoperative, and $14.6 \pm 2.7 \mathrm{mmHg}$ at the last follow-up visit. The two eyes on AGM before the surgery continued to receive topical AGM until the final visit.

\section{Postoperative Complications}

Postoperative transient vitreous hemorrhage was the most common complication $(46.51 \% ; \mathrm{n}=20)$; this resolved spontaneously in 6 weeks in all eyes. The week-wise postoperative complications are listed in Table 1.

Cystoid macular edema (CME) was noted in 9 cases at different follow-up visits and required minimal intervention. CME regressed spontaneously in 3 of 9 eyes; the other six eyes responded well to topical nepafenac $0.1 \%$. A majority $(77.8 \% ; n=7)$ of the eyes that developed postoperative CME had a history of trauma.

The mean presenting visual acuity preoperatively was $1.70 \pm 0.50$ (range 0.3-2.8) $\log$ MAR (Snellen equivalent 20/40 to PL PR accurate) and at 6 weeks postoperative improved significantly $(\mathrm{P}$ value $<0.0001)$

Table I Postoperative Complications (D - Day; W - Week; M - Months)

\begin{tabular}{|l|c|c|c|c|}
\hline Postop Complications & DI & W 2 & W 6 & Last Visit (Mean 28 $\pm \mathbf{8}$ M) \\
\hline None & $51.16 \%$ & $74.41 \%$ & $79.05 \%$ & $90.35 \%$ \\
Choroidal detachment & $2.33 \%$ & $2.33 \%$ & $0 \%$ & $0 \%$ \\
Vitreous haemorrhage & $46.51 \%$ & $11.63 \%$ & $2.33 \%$ & $0 \%$ \\
Cystoid macular edema & $0 \%$ & $9.30 \%$ & $11.63 \%$ & $2.66 \%$ \\
Glaucomatous Disc & $0 \%$ & $2.33 \%$ & $2.33 \%$ & $2.33 \%$ \\
Subconjunctival haptic exposure & $0 \%$ & $0 \%$ & $4.66 \%$ & $4.66 \%$ \\
Total & $43(100.00 \%)$ & $43(100.00 \%)$ & $43(100.00 \%)$ & $43(100.00 \%)$ \\
\hline
\end{tabular}




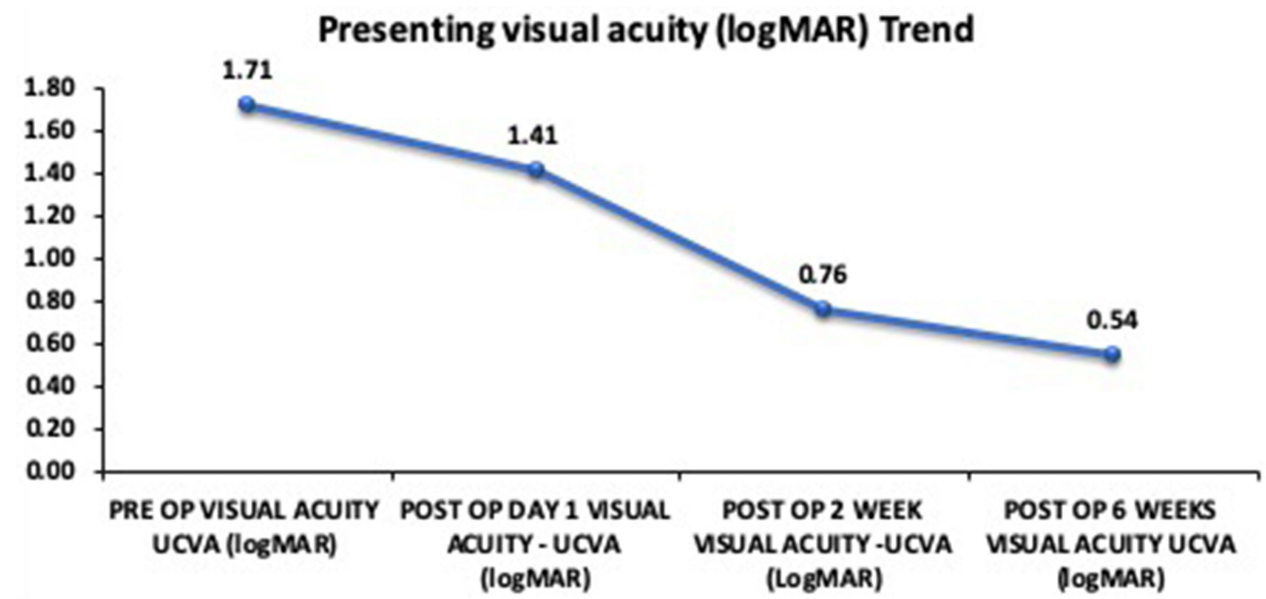

Figure 2 The uncorrected visual acuity (UCVA) trend (logMAR) shows a gradual improvement in vision over 6-week period mostly attributed to vitreous hemorrhage.

to $0.54 \pm 0.28$ (range $0-1.2$ ) $\log$ MAR (Snellen equivalent 20/20 to 20/320) (Figure 2). The improvement was maintained at the final refractive assessment visit $(0.53$ $\pm 0.33 \log$ MAR; $p<0.0001)$ at 24.35 ( \pm 6.71) weeks. The mean residual spherical equivalent spectacle correction at that visit was $+0.74 \pm 1.2 \mathrm{D}$ (range $-3.25 \mathrm{D}$ to $+3 \mathrm{D}$ ) (cylinder $-1.6 \pm 1.5 \mathrm{D}$ at $84 \pm 50^{\circ}$ ). The mean bestcorrected vision at the last visit $(0.22 \pm 0.24 \log$ MAR; Snellen equivalent 20/32) showed a significant improvement $(\mathrm{p}=0.0003)$ as well.

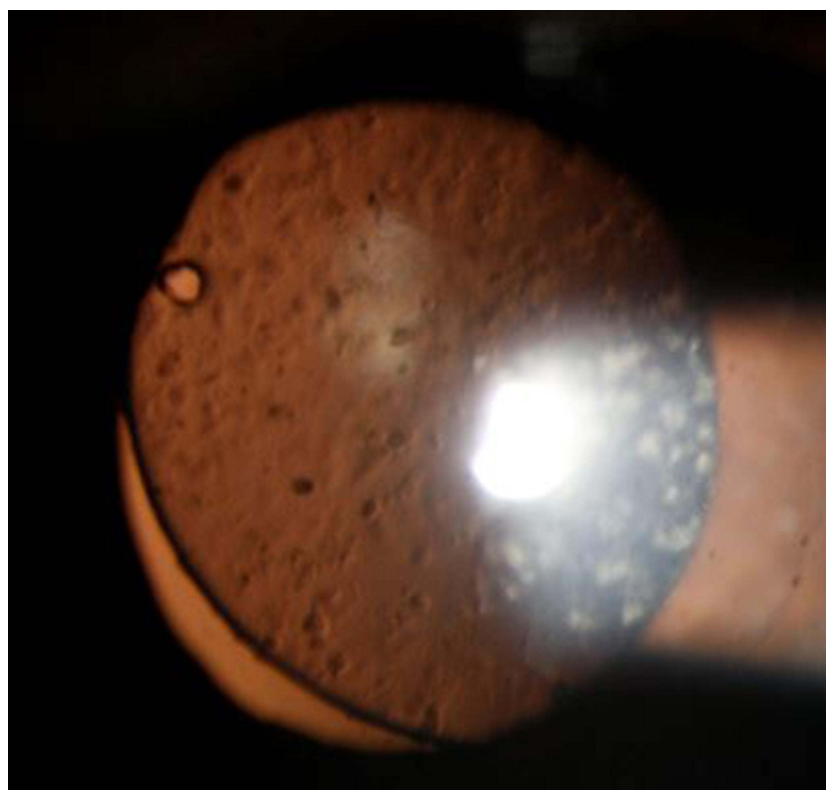

Figure 3 Lens deposits: Slit lamp capture on retro illumination shows cellular deposits on the surface of a scleral fixated intraocular lens 2 wks post-surgery. Aphakia was caused by an open globe injury.
Punctate cellular deposits on the IOL surface were observed in $18.6 \%$ of cases at two weeks (Figure 3). As documented in subsequent clinical photos, the density of the deposits did not show worsening; these deposits did not affect the visual acuity. The exact cause of these deposits could not be elucidated, but $87.5 \%$ of IOLs with deposits had a preoperative history of intense ocular inflammation (endophthalmitis, turbulent primary surgery, and trauma).

At the 6th-month follow-up visit, the IOL position on the clinical photographs as graded by the independent observers was central $(\mathrm{p}=0.001$; kappa 0.411 ; Observer $1-93 \%$, Observer $2-88.4 \%$ ). Likewise, the IOL was placed parallel to the iris plane on UBM in most cases ( $\mathrm{p}<0.0001$; kappa 0.762; Observer $1-88.4 \%$, Observer 2-86\%). There was no clinically evident tilt in the latter part of the year in all eyes, but for one - the lens tilt was noted concurrently with the penetrating keratoplasty graft failure that occurred at the seventh postoperative month; possibly due to hypotony.

The mean duration of surgery in our study cohort of 43 patients was $31.81 \pm 9.85$ minutes (range 17-54 mins). The mean follow-up of the cohort was $28 \pm 8$ months.

\section{Discussion}

Sulcus fixation of IOL with permanent incarceration of haptics in a scleral tunnel parallel to the limbus is less technically demanding and stabilizes the IOL in the posterior chamber without difficult suturing procedures. ${ }^{5,10}$ The basic technique involves two basic steps of haptic externalization and incarceration in the scleral tissue. Several modifications to these steps have been suggested. These include the use of smallbore cannulas/needles instead of bulky forceps for haptic 
externalization; ${ }^{9,13-17}$ and improvement of IOL haptic stability with partial-thickness scleral flaps, fibrin glue, Y-shaped scleral incision, haptic flanging and suturing the externalized haptic to the sclera after docking. . $^{6,11,18-20}$

We made a novel adaptation of haptic exteriorization and Intra scleral haptic fixation. This was the largest prospective study examining the safety and stability of a modified trans scleral IOL fixation technique (medline search) to the best of our knowledge. The study included patients of all age groups, including children $(9.3 \%$ children), and eyes with open globe injuries (32.6\%).

Externalization and incarceration into the scleral pockets of a shorter haptic length with a $60-90^{\circ}$ bend at the tip stabilized the axial position of the IOL. They minimized the risk of IOL tilt/decentration. Cauterizing the haptic tip to a flange as popularized by Yamane et al is a similar modification in this direction. Still, the greater freedom of haptic readjustment to recenter the IOL with our technique is an advantage. ${ }^{8}$ Maintaining a taut globe while tucking was crucial in facilitating a bend at the haptic tip. Using a silicone stopper to temporarily immobilize the leading haptic while working on the trailing haptic, as described by Baskaran et al, ${ }^{21}$ prevented wound leak and collapse of the globe. To minimize manipulations, the haptic-tip was negotiated into the lumen of the needle in the anterior chamber. The silicone stopper obviated the need for an assistant.

We believe that a trans-conjunctival approach ${ }^{5,14,15,22-25}$ would make the surgery minimally invasive (in contrast to our method that needed conjunctival peritomy) with faster postoperative recovery and the conjunctiva saved for future filtration surgery if any. But a transconjunctival approach would require corneal incisions and foldable IOLs. In our hands, the transconjunctival approach (in an open globe injury eye) led to a ballooning of conjunctiva and Tenons, obscuring the scleral pocket from view soon after making the sclerotomy. The puffed-out conjunctiva had to be dissected to get the scleral pocket back in sight. Hence, the transconjunctival approach should be reserved only for eyes with a densely adherent conjunctiva at the haptic docking region of the eye.

Vitreous hemorrhage, intrinsic to trans scleral incisions ${ }^{8,9,13-17,23,24,26}$ and ciliary sulcus-based sclerotomies $^{14}$ was noted in $46.5 \%$ of our patients; this was incidentally high for SFIOL surgery. Vitreous hemorrhage could be attributable to incising the pre-existing sclero-corneal tunnel all over again $(41.8 \%)$, the relative inexperience of the surgeons in fashioning sclero-corneal tunnels, and operating on eyes with a preceding history of trauma $(32.6 \%)$.

Cystoid macular edema, seen in this series, was mostly associated with prior history of trauma (77.8\%). This is not uncommon, though, , $^{11,14-16,18,23,27-29}$ we wonder if a preoperative topical anti-inflammatory drug would have made any difference.

Postoperative haptic exposure is a feared complication of this procedure. ${ }^{16,20,30}$ To prevent this complication, we took care to dock the bent haptic deep in the scleral pocket and drape the scleral incision with the conjunctiva. In this series, we encountered two postoperative subconjunctival haptic show instances; both were open globe injuries. Since the haptic did not erode the overlying conjunctiva, these eyes were kept under close observation; the wounds and the IOL were stable at their last visit, 28 months post-surgery. Only in one case, there was haptic dislocation that required IOL repositioning; this patient had a thinned-out sclera.

In this series, there were no iris-related issues (UBM confirmation) and this could be the determining reason behind absence of IOP-related complications ${ }^{7,11,14,16,17,28}$ and optic capture. $^{8,9,13,16,23,29}$ The choroidal detachment secondary to transient hypotony was self-limiting. $7,11,14,17,28$

Based on previous reports of scleral fixation surgery, we had tried to correct the myopic shift by reducing the IOL power by one diopter. ${ }^{12}$ But as the IOL plane was found to be coinciding with the plane of in-the-bag IOL on UBM, and the average residual postoperative refractive correction was close to one diopter, we do not recommend correcting for the myopic shift for our technique. We used photographic assessments by independent examiners, and in their judgments, the IOL centration was good.

The limitation of this study is the lack of a control group. The 1-year UBM and OCT data were not available as the safety and efficacy endpoints were set at six months. However, the absence of vision drop and lens subluxation at one year may represent the continued efficacy of the procedure. Compared to the described methods in the literature, this technique is invasive in terms of the extent of the peritomy, length of the corneoscleral section, and dependency on rigid IOL. But they are standard for small incision cataract surgery. Complications such as postoperative vitreous cavity hemorrhage and the lens deposits were higher than the reported literature, reportedly uncommon in experienced hands. We believe the prospective nature of the study allowed detection of these subtle complications that would otherwise have been missed in a retrospective study. The study's strength is the long-term 
(mean follow-up of $28 \pm 8$ months) safety and stability outcome. The key defining elements of our technique modification make this surgery reliable, efficient, reproducible, and well suited for surgeons in training for small incision cataract surgery, as a sclero-corneal tunnel and use of rigid intraocular lens is a prerequisite for this technique. Training the resident surgeons with this technique may empower them to manage their aphakia complication on the table, thereby reducing the burden of further secondary interventions.

\section{Funding}

Hyderabad Eye Research Foundation.

\section{Disclosure}

The authors report no conflicts of interest in this work.

\section{References}

1. Fletcher A, Vijaykumar V, Selvaraj S, Thulasiraj RD, Ellwein LB. The Madurai intraocular lens study. III. Visual functioning and quality of life. Am J Ophthalmol. 1998;125(1):26-35. doi:10.1016/S00029394(99)80231-1

2. Monteiro M, Marinho A, Borges S, Ribeiro L, Correia C. Scleral fixation in eyes with loss of capsule or zonule support. $J$ Cataract Refract Surg. 2007;33(4):573-576. doi:10.1016/j.jcrs.2006.10.073

3. Madhivanan N, Sengupta S, Sindal M, Nivean PD, Kumar MA, Ariga M. Comparative analysis of retropupillary iris claw versus scleral-fixated intraocular lens in the management of post-cataract aphakia. Indian JOphthalmol. 2019;67(1):59-63. doi:10.4103/ijo.IJO_326_18

4. Evereklioglu C, Er H, Bekir NA, Borazan M, Zorlu F. Comparison of secondary implantation of flexible open-loop anterior chamber and scleral-fixated posterior chamber intraocular lenses. $J$ Cataract Refract Surg. 2003;29(2):301-308. doi:10.1016/S0886-3350(02)01526-2

5. Gabor SGB, Pavlidis MM. Sutureless intrascleral posterior chamber intraocular lens fixation. J Cataract Refract Surg. 2007;33 (11):1851-1854. doi:10.1016/j.jcrs.2007.07.013

6. Agarwal A, Kumar DA, Jacob S, Baid C, Agarwal A, Srinivasan S. Fibrin glue-assisted sutureless posterior chamber intraocular lens implantation in eyes with deficient posterior capsules. $J$ Cataract Refract Surg. 2008;34(9):1433-1438. doi:10.1016/j.jcrs.2008.04.040

7. Yamane S, Inoue M, Arakawa A, Kadonosono K. Sutureless 27 $\mathrm{G}$ needle guided intrascleral intraocular lens implantation with lamellar scleral dissection. Ophthalmology. 2014;121(1):61-66. doi:10.1016/j.ophtha.2013.08.043

8. Yamane S, Sato S, Maruyama-Inoue M, Kadonosono K. Flanged intrascleral intraocular lens fixation with double-needle technique. Ophthalmology. 2017;124(8):1136-1142. doi:10.1016/j. ophtha.2017.03.036

9. Scharioth GB, Prasad S, Georgalas I, Tataru C, Pavlidis M. Intermediate results of sutureless intrascleral posterior chamber intraocular lens fixation. $J$ Cataract Refract Surg. 2010;36:254-259.

10. Sindal MD, Nakhwa CP, Sengupta S. Comparison of sutured versus sutureless scleral-fixated intraocular lenses. J Cataract Refract Surg. 2016;42(1):27-34. doi:10.1016/j.jcrs.2015.09.019

11. Shekhawat N, Goyal K. Sutureless glueless intrascleral fixation of posterior chamber intraocular lens: boon for aphakic. Indian J Ophthalmol. 2017;65(12):1454-1458. doi:10.4103/ijo.IJO_620_17
12. Suto C, Hori S, Fukuyama E, Akura J. Adjusting intraocular lens power for sulcus fixation. J Cataract Refract Surg. 2003;29 (10):1913-1917. doi:10.1016/S0886-3350(03)00250-5

13. Takayama K, Akimoto $M$, Taguchi H, Nakagawa S, Hiroi $K$. Transconjunctival sutureless intrascleral intraocular lens fixation using intrascleral tunnels guided with catheter and 30-gauge needles. $\mathrm{Br}$ J Ophthalmol. 2015;99(11):1457-1459. doi:10.1136/bjophthalmol-2014306579

14. Wilgucki JD, Wheatley HM, Feiner L, Ferrone MV, Prenner JL. One year outcomes of eyes treated with a sutureless scleral fixation technique for intraocular lens placement or rescue. Retina. 2015;35 (5):1036-1040. doi:10.1097/IAE.0000000000000431

15. Abbey AM, Hussain RM, Shah AR, Faia LJ, Wolfe JD, Williams GA. Sutureless scleral fixation of intraocular lenses: outcomes of two approaches. The 2014 Yasuo Tano memorial lecture. Graefes Arch Clin Exp Ophthalmol. 2015;253(1):1-5. doi:10.1007/s00417-014-2834-9

16. Bonnell AC, Mantopoulos D, Wheatley HM, Prenner JL. Surgical technique for sutureless intrascleral fixation of a 3-piece intraocular lens using a 30-gauge needle. Retina. 2019;39(1):S13-S15. doi:10.1097/IAE.0000000000001889

17. Kelkar AS, Fogla R, Kelkar J, Kothari AA, Mehta H, Amoaku W. Sutureless 27-gauge needle-assisted transconjunctival intrascleral intraocular lens fixation: initial experience. Indian $J$ Ophthalmol. 2017;65(12):1450-1453. doi:10.4103/ijo.IJO_659_17

18. Ohta T, Toshida H, Marukami A. Simplified and safe method of sutureless intrascleral posterior chamber intraocular lens fixation: y Fixation technique. J Cataract Refract Surg. 2014;40(1):2-7. doi:10.1016/j.jcrs.2013.11.003

19. Liu S, Cheng S. Modified method of sutureless intrascleral posterior chamber intraocular lens fixation without capsular support. Eur J Ophthalmol. 2013;23(5):732-737. doi:10.5301/ejo.5000281

20. Saleh M, Heitz A, Bourcier T, et al. Sutureless intrascleral intraocular lens implantation after ocular trauma. J Cataract Refract Surg. 2013;39(1):81-86. doi:10.1016/j.jcrs.2012.08.063

21. Baskaran P, Ganne P, Bhandari S, Ramakrishnan S, Venkatesh R, Gireesh P. Extraocular needle- guided haptic insertion technique of scleral fixation intraocular lens surgeries (X-NIT). Indian J Ophthalmol. 2017;65(8):747-750. doi:10.4103/ijo.IJO_296_17

22. Thanos A, Lau-Sickon LK, Wolfe JD, Hassan TS. Three port sutureless posterior chamber intraocular lens intrascleral fixation: a novel approach. Retina. 2019;39(1):S16-S20. doi:10.1097/IAE.0000 000000001925

23. Kawaji T, Sato T, Tanihara H. Sutureless intrascleral intraocular lens fixation with lamellar dissection of scleral tunnel. Clin Ophthalmol. 2016;10:227-231. doi:10.2147/OPTH.S101515

24. Rajesh Prabu V, Kapoor S, Udayakumar S, Gupta P. Retrospective study of visual outcomes and complications after sutureless, flapless, and glueless intrascleral fixation of posterior chamber intraocular lens in children and young adults. J Pediatr Ophthalmol Strabismus. 2017;54(6):381-386. doi:10.3928/01913913-20170703-10

25. Prasad S. Transconjunctival sutureless haptic fixation of posterior chamber IOL: a minimally traumatic approach for IOL rescue or secondary implantation. Retina. 2013;33(3):657-660. doi:10.1097/ IAE.0b013e31827b6499

26. Totan Y, Karadag R. Two techniques for sutureless intrascleral posterior chamber IOL fixation. J Refract Surg. 2013;29(2):90-94. doi:10.3928/1081597X-20130117-02

27. Walsh MK. Sutureless trocar-cannula-based transconjunctival flanged intrascleral intraocular lens fixation. Retina. 2017;37(11):2191-2194. doi:10.1097/IAE.0000000000001593

28. Zhang Y, He F, Li Q, Wang Z. Modified technique for intrascleral fixation of posterior chamber intraocular lens without scleral flaps. $J$ Cataract Refract Surg. 2017;43(2):162-166. doi:10.1016/j. jers.2016.10.029 
29. Kumar DA, Agrawal A, Agrawal A, Prakash G, Jacob S. Glued intraocular lens Implantation for eyes with deceptive capsules: a retrospective analysis of anatomical and functional outcome. Saudi J Ophthalmol. 2011;25(3):245-254. doi:10.1016/j. sjopt.2011.04.001
30. Kumar DA, Agarwal A, Packiyalakshmi S, Jacob S, Agarwal A. Complications and visual outcomes after glued foldable intraocular lens implantation in eyes with inadequate capsules. J Cataract Refract Surg. 2013;39(8):1211-1218. doi:10.1016/j.jcrs.2013.03.004

\section{Publish your work in this journal}

Clinical Ophthalmology is an international, peer-reviewed journal covering all subspecialties within ophthalmology. Key topics include: Optometry; Visual science; Pharmacology and drug therapy in eye diseases; Basic Sciences; Primary and Secondary eye care; Patient Safety and Quality of Care Improvements. This journal is indexed on PubMed

Submit your manuscript here: https://www.dovepress.com/clinical-ophthalmology-journal
Central and CAS, and is the official journal of The Society of Clinical Ophthalmology (SCO). The manuscript management system is completely online and includes a very quick and fair peer-review system, which is all easy to use. Visit http://www.dovepress.com/ testimonials.php to read real quotes from published authors. 